\title{
ChemComm
}

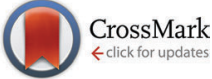

Cite this: Chem. Commun., 2016, 52, 3639

Received 11th December 2015, Accepted 27th January 2016

DOI: $10.1039 / c 5 c c 10222 g$

www.rsc.org/chemcomm

\section{Gate-opening effect in ZIF-8: the first experimental proof using inelastic neutron scattering $\dagger$}

\author{
M. E. Casco, ${ }^{\text {a } Y . ~ Q . ~ C h e n g, ~}{ }^{\text {b }}$ L. L. Daemen, ${ }^{\text {b }}$ D. Fairen-Jimenez, ${ }^{c}$ \\ E. V. Ramos-Fernández, ${ }^{a}$ A. J. Ramirez-Cuesta*b and J. Silvestre-Albero*a
}

The gate-opening phenomenon in ZIFs is of paramount importance to understand their behavior in industrial molecular separations. Here we show for the first time using in situ inelastic neutron scattering (INS) the swinging of the $-\mathrm{CH}_{3}$ groups and the imidazolate linkers in the prototypical ZIF-8 and ZIF-8@AC hybrid materials upon exposure to mild $\mathrm{N}_{2}$ pressure.

Swing- and gate-opening effects, nicely described by Kitagawa et al., are one of the most exciting phenomena in materials science providing unusual properties to the materials such as molecular selectivity and sensing ability. ${ }^{1,2}$ This phenomenon is characteristic of inorganic solids with certain structural flexibility upon interaction with an external stimuli, e.g. pressure, temperature, light, electric or magnetic field, etc. Structural flexibility has been widely applied for a wide range of applications (e.g. gas separation). Among the different inorganic materials with structural flexibility, metal-organic frameworks (MOFs) are highly exciting due to the combination of high specific surface area, well-defined porous structure, and large versatility in terms of composition (and hence surface chemistry). Therefore, understanding the structural flexibility in MOFs is of paramount importance to design/tailor new structures with improved properties (e.g. molecular selectivity).

Although the structural flexibility in MOF materials has been traditionally associated with important changes in the unit cell and pore volume due to the associated structural expansion/contraction (e.g. breathing phenomena with $c a$. $40 \%$ variation in the case of

\footnotetext{
${ }^{a}$ Laboratorio de Materiales Avanzados, Departamento de Quimica Inorgánica-Instituto Universitario de Materiales, Universidad de Alicante, Ctra. San Vicente-Alicante $s / n$, E-03690 San Vicente del Raspeig, Spain. E-mail: joaquin.silvestre@ua.es

${ }^{b}$ Oak Ridge National Laboratory, Chemical and Engineering Materials Division, 1 Bethel Valley Road, Oak Ridge, Tennessee 37831, USA.

E-mail: timmy.ramirez.cuesta@gmail.com

${ }^{c}$ Department of Chemical Engineering and Biotechnology, University of Cambridge, Pembroke Street, Cambridge CB2 3RA, UK

$\dagger$ Electronic supplementary information (ESI) available: A detailed description of the synthesis procedure for the ZIF-8 and hybrid material ZIF-8@AC, material characterization ( $\mathrm{N}_{2}$ adsorption isotherms, SEM and XRD patterns), DFT calculations and gif animations for the gate-opening motions. See DOI: 10.1039/c5cc10222g
}

MIL-53), this is not always true in the case of some zeolitic imidazolate framework (ZIFs) materials. ${ }^{3}$ ZIFs are a relatively new class of MOFs that maintain their excellent adsorption properties, but with a higher chemical and thermal stability. Despite its rigid zeolitic topology, sodalite (SOD) materials like ZIF-8, ZIF-7, ZIF-65, ZIF-90 and ZIF-91 exhibit some characteristic swing-effect phenomena when exposed to an external gas pressure (e.g. $\mathrm{N}_{2}$ or $\mathrm{O}_{2}$ ) or when subjected to high mechanical stress (for example above $1.47 \mathrm{GPa}$ in a diamond anvil cell). ${ }^{3-6}$ Indeed, $\mathrm{N}_{2}$ gas-adsorption isotherms in ZIF-8 exhibit a characteristic two-step adsorption in the relative pressure range from $p / p_{0} \sim 0.01$ to 0.1 , sometimes associated with a hysteresis loop (see Fig. S1, ESI $\dagger$ ). Despite the large number of experimental and theoretical studies dealing with ZIF-8, the nature of this structural flexibility is still under debate. Whereas some authors have attributed these two steps to the reorganization of the adsorbed $\mathrm{N}_{2}$ molecules during the adsorption process, rejecting the presence of structural changes, ${ }^{6}$ grand canonical Monte Carlo (GCMC) simulations have anticipated the presence of a gas-induced rotation of the imidazolate linkers upon pressure but without any appreciable change in the crystal dimensions. ${ }^{3}$ Despite these premises, recent experimental and theoretical studies have suggested that the structural flexibility in ZIF-8 goes beyond the gas-induced gate-opening effect, and an expansion of the main cavity of ZIF-8 is required to understand the adsorption behavior. ${ }^{4}$

To gain greater insight into the nature of the structural flexibility of ZIF-8 and to understand its adsorption behavior, we have evaluated the structural flexibility of ZIF-8 upon gas exposure by performing in situ inelastic neutron scattering (INS) experiments. INS is especially sensitive to the dynamics of hydrogen and hydrogen-bearing groups due to the relatively large incoherent neutron cross-section of hydrogen compared to other atomic nuclei in ZIFs. INS is therefore a powerful tool for the evaluation of the dynamics of the methyl group on the imidazolate linkers. ${ }^{7-9}$ To evaluate further the possible structural deformations (expansion and contraction effects) upon gas exposure, the gate-opening effect has been evaluated in ZIF-8 nanocrystals nucleated and grown in the restricted nanospace of specifically designed 


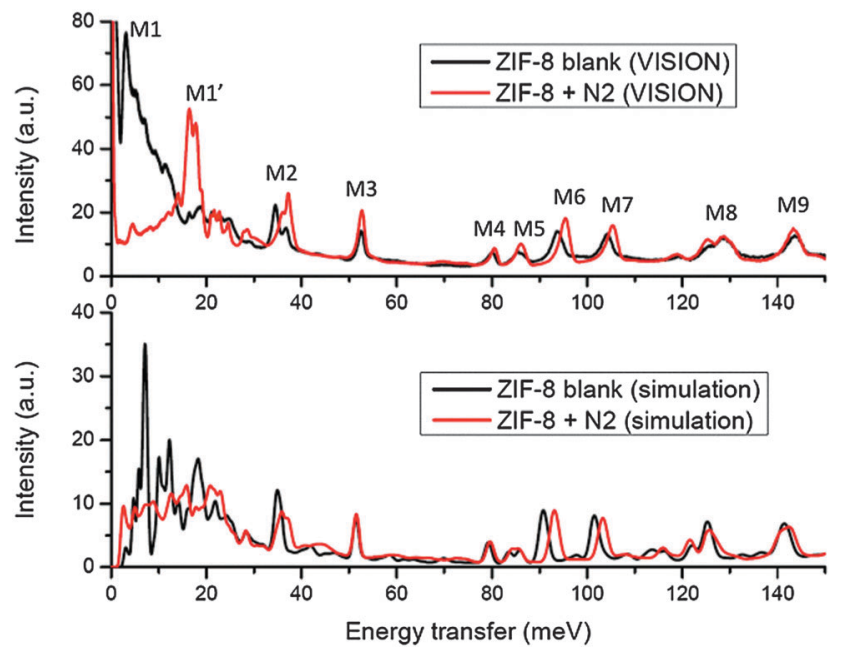

Fig. 1 Measured (upper panel) and simulated (lower panel) INS spectra of blank ZIF-8 and ZIF-8@ $\mathrm{N}_{2}$

nanoporous carbon materials. The confinement of MOF nanocrystals in the cavities of carbon nanomaterials (hybrid MOF@AC) has been recently proposed by some of us as a promising approach to improve the mechanical properties of these systems, provided that confined nanocrystals can retain the structural and adsorptive properties of the parent MOF. ${ }^{10}$

Fig. 1 (upper panel) shows the high-resolution inelastic neutron scattering (INS) measurements performed on the VISION spectrometer at the Spallation Neutron Source at Oak Ridge National Laboratory. The INS spectrum covers an energy transfer range up to $150 \mathrm{meV}$, and shows the most relevant rotational and vibrational modes of the zeolitic-imidazole framework. The INS spectrum of ZIF-8 can be divided into three regions: (i) the low energy transfer region (0-30 $\mathrm{meV})$, which reveals the dynamics of the framework opening and contains the methyl torsions and the vibrational modes of the imidazole linkers, (ii) the middle energy region (30-70 meV), which contains ring deformation modes, and (iii) the higher-energy region (above $70 \mathrm{meV}$ ), which contains the methylene wagging, twisting, rocking and $\mathrm{C}-\mathrm{H}$ bending modes. A more detailed explanation of these peaks (M1 to M9) will be given later.

In ZIF-8, the 2-methylimidazole groups are linked to $\mathrm{Zn}$ cations by way of two $\mathrm{N}$ atoms in the imidazole ring. The methyl group is attached to $\mathrm{C} 2-$ the $\mathrm{C}$ atom between the two $\mathrm{N}$ atoms. The experimental INS spectrum of pure ZIF-8 contains an intense band at $\sim 3.08 \mathrm{meV}$ that can be unambiguously attributed to the second rotational level of the methyl group from the imidazolate linkers (rotational transition $J=0 \rightarrow J=2$ ). ${ }^{11}$ The asymmetry of this peak suggests that additional modes may be present. A close examination reveals the presence of two shoulders at $\sim 5.03 \mathrm{meV}$ and $\sim 7.02 \mathrm{meV}$, which must be attributed to additional methyl torsional, translational and/or rotational modes. The ineslastic rotational constant of methane as a free rotor is $B_{\mathrm{CH}_{4}}=h / 2 \Theta \sim 0.655 \mathrm{meV}$ (ground state rotational level $J=0 \rightarrow 1$ ), whereas the second rotational energy at $\sim 3.08 \mathrm{meV}$ is consistent with a very low rotational barrier. Consequently, the peak appearing at $\sim 3.08 \mathrm{meV}$ in ZIF-8 suggests a weak interaction between nearest-neighbor methyl groups from the imidazolate linkers, in agreement with their structural arrangement.

The spectrum changes drastically after exposure of the sample to $80 \mathrm{kPa}(0.8 \mathrm{bar})$ of $\mathrm{N}_{2}$ at $77 \mathrm{~K}$ for $10 \mathrm{~min}$, followed by cooling to $10 \mathrm{~K}$ before collecting another INS spectrum. As can be observed in Fig. 1 (upper panel), the $\sim 3.08 \mathrm{meV}$ band attributed to the free rotation of the $-\mathrm{CH}_{3}$ group completely vanishes in the presence of nitrogen, whereas a new band emerges at a higher energy transfer with maxima at $\sim 16.4 \mathrm{meV}$ and $\sim 17.7 \mathrm{meV}$. The shift observed in the main band of the ZIF-8, upon exposure to $\mathrm{N}_{2}$, clearly suggests a change in the molecular environment of the methyl groups. This behavior is consistent with increased steric hindrance in the methyl rotations caused by a swinging of the imidazolate rings - a behavior confirmed by the simulations described below. Upon rotation of the imidazolate rings after $\mathrm{N}_{2}$ incorporation in the pores, there is a larger intermolecular interaction of the methyl groups in a cavity. This finding constitutes the first in situ experimental evidence of the rotation of the imidazolate rings in ZIF-8 upon exposure to $\mathrm{N}_{2}$. Interestingly, these changes in the low energy region, where the dynamics of the framework opening are reflected, are not accompanied by important changes in the vibrational and rotational modes in the middle and high-energy region. Indeed, both spectra are rather similar, with only minor shifts of certain peaks. This suggests the absence of large structural deformations and/or expansions. Increased strength in the rotation of the methyl group in ZIF-8 after benzene dosing was also observed by Kolokolov et al. using ${ }^{2} \mathrm{H}$ NMR. However, activation barriers obtained from ${ }^{2} \mathrm{H}$ NMR are relatively larger than the ones obtained from neutron scattering at liquid helium temperature (e.g. rotational energy in the guest-free material is $\sim 13.47 \mathrm{meV}$, from ${ }^{2} \mathrm{H}$ NMR, and $\sim 3.08 \mathrm{meV}$, from INS). ${ }^{12}$

To confirm our interpretation of the INS results, DFT calculations were performed using CASTEP. ${ }^{13}$ Fig. 2 shows the relaxed configurations of ZIF-8 before and after $\mathrm{N}_{2}$ dosing. The rotation of the methyl groups and the swinging of the imidazolate rings associated with the gate opening can be clearly seen by comparing the market areas in (a) and (b) (better illustrations including gif animations of the gate-opening can be found in Fig. S2-S12, $\mathrm{ESI} \dagger$ ). The market interatomic distance in (c) and (d) increases from $2.9 \AA$ to $3.4 \AA$ due to the gate opening.

The simulated INS spectra with and without $\mathrm{N}_{2}$ are plotted in Fig. 1 (lower panel). In general the simulations perfectly reproduce the main features of the INS experimental spectra over the whole energy transfer region evaluated. This includes not only the peak positions in the pure ZIF-8 material, but also the shift of certain peaks upon $\mathrm{N}_{2}$ dosing. The excellent match between the experiments and simulations, which can only be achieved when the swinging of the methyl-imidazolate linkers (i.e. gate-opening) is considered in the simulation, demonstrates unambiguously this effect in ZIF-8. DFT calculations allow us to assign the peaks observed in the experiments and to gain additional insight not directly available from the experimental data. In this sense, the most intense peak for methyl rotation is at $\sim 7 \mathrm{meV}$ in the simulation of ZIF-8, with a number of weaker 
(a)

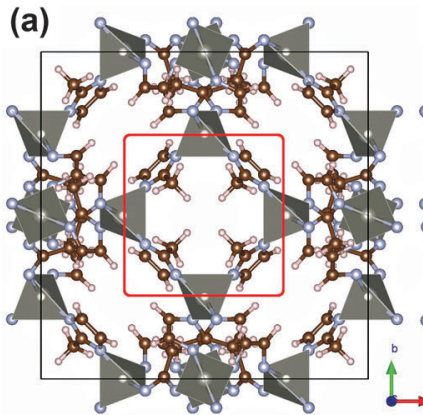

(c)

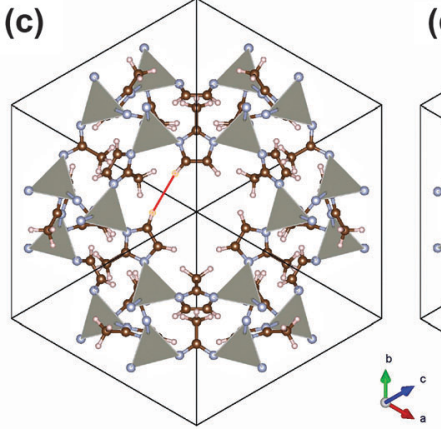

(b)

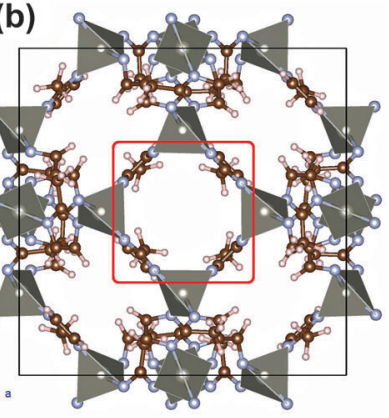

(d)

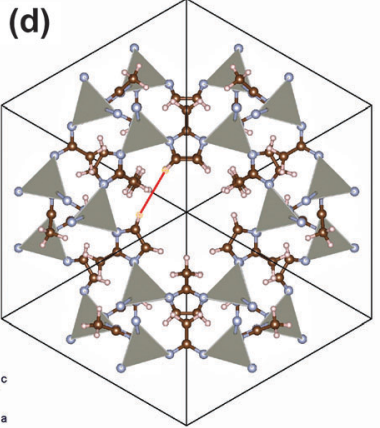

Fig. 2 Structure of blank ZIF-8 viewing from the axis direction (a) and the diagonal direction (c), as well as the corresponding structure of ZIF- 8 loaded with $\mathrm{N}_{2}$, (b) and (d). For clarity the $\mathrm{N}_{2}$ molecules are not shown.

peaks in the energy range of $0-20 \mathrm{meV}$ (see Fig. 1). A careful examination of these vibrational modes shows that the peak around $7 \mathrm{meV}$ correspond to a highly synchronized rotational mode involving multiple methyl groups in a cavity, whereas other weaker peaks (appearing at a higher energy transfer) can be attributed to local individual methyl torsional motion that is probably coupled to ZIF-8 framework modes (see Fig. S2, ESI $\dagger$ ). Torsional and vibrational modes are extremely sensitive to the exact molecular environment of the moiety performing the rotational motion. These large amplitude modes are notoriously difficult to predict with great accuracy. Indeed the computational centers these modes around $7 \mathrm{meV}$, whereas the experimental data reveals them closer to $3 \mathrm{meV}$. The assignment is not in doubt, however. The incorporation of $\mathrm{N}_{2}$ opens the gate, swings the methyl-imidazolate linkers, and thus changes the local potential energy landscape, so that highly synchronized torsional modes (and therefore the intense peak at around $7 \mathrm{meV}$ ) must be largely suppressed. Although this effect is indeed observed in our simulation, experimental measurements show a shift of the main peak for methyl torsion after gate opening to $\sim 17 \mathrm{meV}$. This result suggests that the methyl torsional modes should now synchronize at a significantly higher energy. Although simulations suggest a slight enhancement in intensity at around $20 \mathrm{meV}$, a well-defined peak cannot be observed. This discrepancy could be attributed to the way the partial occupancy of nitrogen was treated when setting up the simulation, which inevitably reduced the global symmetry, and thus may have had an adverse impact on the formation of the synchronized mode.

In addition to the methyl torsion, some other modes are also seen to be slightly affected by the $\mathrm{N}_{2}$ dosing and gate opening, as predicted by DFT. Specifically, the M2 mode at around $35 \mathrm{meV}$, the out-of-plane bending mode of the methyl groups relative to the imidazolate rings, exhibits a slight change in the relative intensity of the different contributions; M6 and M7 bands, attributed to the out-of-plane bending mode of the two $\mathrm{C}-\mathrm{H}$ bonds in the imidazolate rings, with the two $\mathrm{C}-\mathrm{H}$ bonds in the same imidazolate ring moving in-phase (symmetrical) and anti-phase (asymmetrical), respectively, also exhibit a slight shift. No additional changes or shifts could be observed for the other rotational and vibrational modes. Apparently, M2, M6 and $\mathrm{M} 7$ are more sensitive to $\mathrm{N}_{2}$ dosing (Fig. 1). As they are all out-of-plane modes, it can be inferred that the imidazolate rings after gate opening becomes stiffer and more confined along the out-of-plane direction, whereas within the plane they are less affected. This is quite reasonable and in fact expected from the nature of the gate opening process and the positions of the $\mathrm{N}_{2}$ molecules. Pictures showing all the above-mentioned vibrational modes can be found in Fig. S2-S10 (ESI $\dagger$ ).

To investigate further the structural flexibility issue, small nanocrystals of ZIF-8 have been nucleated and grown in the nanocavities of specially designed activated carbons (petroleumpitch derived activated carbons-AC). ${ }^{14}$ As shown in Fig. S13 (ESI $\dagger)$, hybrid MOF@AC $x(x=\mathrm{wt} \%$ carbon) samples preserve the characteristic XRD pattern of the parent MOF, independently of the host-guest ratio. Textural properties of the pure ZIF-8 and the hybrid materials are reported in Table S1 (ESI $\dagger$ ). The textural properties of the pure ZIF-8 sample are very close to those described in the literature, with a BET area above $1600 \mathrm{~m}^{2} \mathrm{~g}^{-1}$ (Type I isotherm) and a total pore volume of $0.72 \mathrm{~cm}^{3} \mathrm{~g}^{-1}$, thus confirming the purely microporous nature of ZIF-8. ${ }^{3}$ In contrast, the AC guest material exhibits an extremely large BET surface area (above $3400 \mathrm{~m}^{2} \mathrm{~g}^{-1}$-type IV isotherm) and a very large total pore volume, as compared to the total micropore volume, thus suggesting the presence of meso and macroporosity able to host the MOF nanocrystals. It is important to highlight that the incorporation of 43 wt\% of ZIF-8 into the AC (sample ZIF-8@AC57) gives rise to a sudden decrease in the BET surface area down to $1700 \mathrm{~m}^{2} \mathrm{~g}^{-1}$. This value is very close to the BET surface area of the guest ZIF-8 and it is unaffected by the host-guest ratio. Previous studies from some of us have anticipated a similar behavior for HKUST-1@AC hybrid materials. ${ }^{10}$ The sudden decrease in the BET surface area and in the total pore volume of the AC sample after growing the ZIF-8 suggest the preferential nucleation and growth of the ZIF nanoscrystals in the nanocavities of the host carbon, although the presence of some MOF in the external surface area cannot be ruled out. In fact, SEM images (Fig. S15, ESI $\dagger$ ) show a perfect distribution of the MOF nanocrystals within the carbon matrix together with some MOFs crystals in the interparticle space and/or in the external surface area (MOFs nanocrystals in the inner cavities of the carbon cannot be observed by SEM). These results are in close agreement with recent studies by some of us where narrow cavities in activated carbons promoted the nucleation and growth of methane hydrate nanocrystals. ${ }^{14}$

As described above, one of the main characteristics of ZIF-8 is the presence of swing-effect phenomena upon exposure to gases (e.g. $\mathrm{N}_{2}$ ) at cryogenic temperatures (Fig. S1, $\mathrm{ESI} \dagger$ ). 


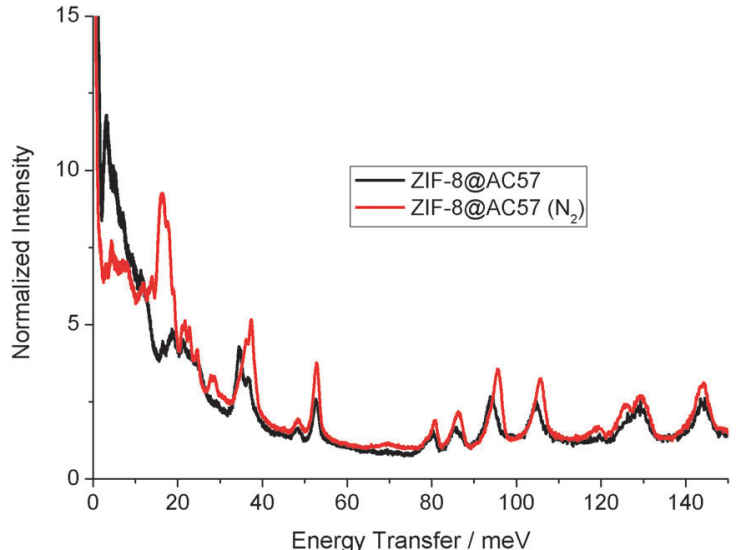

Fig. 3 Inelastic neutron scattering (INS) spectra of ZIF-8@AC57 as prepared and after dosing $80 \mathrm{kPa}$ of $\mathrm{N}_{2}$ at $77 \mathrm{~K}$.

To evaluate the structural flexibility of these nanocrystals in confined nanospace, MOF@AC $x$ samples have been exposed to nitrogen up to $100 \mathrm{kPa}$ at $77 \mathrm{~K}$. As observed in Fig. S14 (ESI $\dagger$ ), the nitrogen adsorption isotherms of the hybrid materials in the pressure range from 0 to $10 \mathrm{kPa}$ highly resembles that of the parent ZIF-8, including the characteristic steps at $\sim 0.73 \mathrm{kPa}$ and $\sim 2.48 \mathrm{kPa}$. In addition, these isotherms exhibit a type IV behavior, mainly at high relative pressures, characteristic of the host carbon. According to the literature, the first step in ZIF-8 might be due to a rearrangement of the $\mathrm{N}_{2}$ molecules due to the presence of strong electrostatic interactions with the ZIF framework (quadrupole-dipole), while the second one is related to the swinging of the imidazolate linkers, i.e. the gate-opening effect. ${ }^{3}$ Taking into account steric hindrance in the cavities of nanoporous carbon materials, the preservation of the swingphenomenon in confined nanospace (including the hysteresis loop) suggests that structural changes in ZIF-8 during $\mathrm{N}_{2}$ adsorption do not go beyond the gas-induced rotation of the imidazolate linker, i.e. large structural expansions/contractions of the ZIF-8 framework can be ruled out, in close agreement with DFT calculations (see ESI $\dagger$ ).

To end up, the hydrid material with the larger proportion of carbon (sample ZIF-8@AC57) has been evaluated using INS. Fig. 3 shows that the INS spectra of the confined ZIF-8 perfectly fits with that of the pure MOF (Fig. 1), although with a lower intensity. The perfect match between these two spectra and the absence of any shift in the XRD pattern (Fig. S13, ESI $\dagger$ ) suggest that the confined nanocrystals retain the structural properties of the original ZIF-8. Exposure to $80 \mathrm{kPa}$ of $\mathrm{N}_{2}$ of the hybrid sample gives rise to the same shift in the low-energy region as compared to the original ZIF-8, thus confirming that the gateopening effect remains unaffected in the confined nanospace. Concerning the middle and high-energy regions, the INS spectra are highly coincident, thus confirming the absence of any significant structural deformation associated with the gate-opening phenomena (even out-of-plane bending modes, M6 and M7, are less affected in confined space).

In summary, we have evaluated for the first time the structural deformations of ZIF-8 using in situ inelastic neutron scattering before and after $\mathrm{N}_{2}$ incorporation at cryogenic temperatures. INS measurements clearly demonstrate that the gas-induced structural deformation in this zeolitic imidazole framework is purely due to the swinging of the imidazolate linker, excluding any additional structural flexibility upon gas exposure. These observations have been confirmed by DFT calculations and via nucleation and growth of ZIF-8 in confined nanospace. The confined ZIF-8 nanoscrystals retain the structural properties (rotational, vibrational and crystallographic properties) of the parent sample, including the swing (gate-opening) effect.

The authors acknowledge financial support from MINECO projects: MAT2013-45008-p and CONCERT Project-NASEMS (PCIN-2013-057). EVRF gratefully acknowledge support from MINECO (Spain) for his Ramón y Cajal grant (RyC-201211427). DFJ thanks the Royal Society (UK) for funding through a University Research Fellowship and Dr Axel Zeitler for interesting discussions. This research benefited from the use of the VISION beamline (IPTS-13608) at ONRL's Spallation Neutron Source and the VirtuES (Virtual Experiments in Spectroscopy) project, (LDRD 7739), which are supported by the Scientific User Facilities Division, Office of Basic Energy Sciences, U.S. Department of Energy, under Contract No. DE-AC0500OR22725 with UT Battelle, LLC.

\section{References}

1 S. Kitagawa and K. Uemura, Chem. Soc. Rev., 2005, 34, 109.

2 G. Férey and C. Serre, Chem. Soc. Rev., 2009, 38, 1380.

3 D. Fairen-Jimenez, S. A. Moggach, M. T. Wharmby, P. A. Wright, S. Parsons and T. Düren, J. Am. Chem. Soc., 2011, 133, 8900.

4 C. O. Ania, E. García-Pérez, M. Haro, J. J. Gutierrez-Sevillano, T. ValdésSolís, J. B. Parra and S. Calero, J. Phys. Chem. Lett., 2012, 3, 1159.

5 S. A. Moggach, T. D. Bennett and A. K. Cheetham, Angew. Chem., Int. Ed., 2009, 48, 7087.

6 K. S. Park, Z. Ni, A. P. Côte, J. Y. Choi, R. D. Huang, F. J. Uribe-Romo, H. K. Chae, M. O'Keeffe and O. M. Yaghi, Proc. Natl. Acad. Sci. U. S. A., 2006, 103, 10186.

7 M. R. Ryder, B. Civalleri, T. D Bennett, S. Henke, S. Rudic, G. Cinque, F. Fernández-Alonso and J.-C. Tan, Phys. Rev. Lett., 2014, 113, 215502.

8 W. Zhou, H. Wu, T. J. Udovic, J. J. Rush and T. Yildirim, J. Phys. Chem. A, 2008, 112, 12602.

9 N. Y. Tan, M. T. Ruggiero, C. Orellana-Tavra, T. Tian, A. D. Bond, T. M. Korter, D. Fairén-Jimenez and J. A. Zeitler, Chem. Commun., 2015, 51, 16037.

10 M. E. Casco, J. Fernández-Catalá, M. Martínez-Escandell, F. Rodríguez-Reinoso, J. M. Ramos-Fernández and J. SilvestreAlbero, Chem. Commun., 2015, 51, 14191.

11 P. C. H. Mitchell, S. F. Parker, A. J. Ramirez-Cuesta and J. Tomkinson, Vibrational Spectroscopy with Neutrons, World Scientific, Singapour, 2005.

12 D. I. Kolokolov, A. G. Stepanov and H. Jobic, J. Phys. Chem. C, 2015, 119, 27512.

13 S. J. Clark, M. D. Segall, C. J. Pickard, P. J. Hasnip, M. J. Probert, K. Refson and M. C. Payne, Z. Kristallogr., 2005, 220, 567.

14 M. E. Casco, J. Silvestre-Albero, A. J. Ramírez-Cuesta, F. Rey, J. L. Jordá, A. Bansode, A. Urakawa, I. Peral, M. Martínez-Escandell, K. Kaneko and F. Rodríguez-Reinoso, Nat. Commun., 2015, 6, 6432. 